\title{
STABILITY ANALYSIS FOR DISCRETE DYNAMICAL MODELS USING MATHEMATICA
}

\author{
ÜNAL UFUKTEPE ${ }^{1}$, BURÇİN KÜLAHÇIOĞLU ${ }^{2}$ \\ ${ }^{1}$ Izmir University of Economics, Department of Mathematics \\ unal.ufuktepe@ieu.edu.tr \\ ${ }^{2}$ Izmir University of Economics, Department of Mathematics \\ burcin.kulahcioglu@ieu.edu.tr
}

\begin{abstract}
Stability is one of the most important concepts in Discrete Dynamical Systems. Behaviour of orbits in the neighbourhood of fixed points can tell much about the behaviour of the system. Although in literature there are some computer codes to find stability types of the fixed points, they are generally lack of non-hyperbolic fixed points for one dimensional models and center manifolds for two dimensional models. We give Mathematica codes for the stability of one dimensional and two dimensional models with non-hyperbolic cases and center manifolds. These codes will be useful for whom dealing with real world problems including population growth, compound interest and annuities, radioactive decay and pollution control,etc.

Keywords: Discrete Dynamical systems, Stability Analysis, Fixed Points, Center Manifold,Mathematica
\end{abstract}

1. Introduction. Dynamical modelling is the study of change and changes take place everywhere in life. As a result dynamical systems have a wide range of application areas in applied science and engineering. With these systems, real life situations can be turned into the language of mathematics. Discrete dynamical systems are described by difference equations (or recurrence relations) so it has mathematical simplicity and amenability to computerization. This encourages researchers to use discrete dynamical models in many disciplines. If one can create a good model for a real life situation, we will be able to predict the future states of the system by simply iterations according to this model. But even the simplest models can exhibit complex behavior in the long run and can go to chaos. Instead of doing large number of iterations, it is something logical if we look at the behavior of orbits near fixed points. Stability Theory is important as itself as well as it serves to the Chaos Theory.

Genetics, population biology, engineering, chemistry, economics are some of the areas that discrete dynamical systems are widely used for modelling. For example for population biology, let $x_{n}$ denote the number of species at time $\mathrm{n}$, then $x_{n+1}$ denotes the number of species one step later that is at time $\mathrm{n}+1$. One can find a pattern between $x_{n}$ and $x_{n+1}$ taking into account birth, death, immigration, and emigration. This will be a one dimension linear discrete dynamical model for population. On the other hand, if she/he adds to the model intra-specific interactions then the new model will be a nonlinear one dimensional model. Furthermore, if interspecific interactions with another species are included, the new system will be two or more dimensional model. 
Mathematica is a very popular computational software program used in many scientific, engineering, mathematical and computing fields. It is a symbolic, numerical and graphical manipulation package [3]. This paper includes Mathematica codes for stability and Center manifolds. These codes will be useful for whom want to do stability analysis of a system without getting lost in long proofs and theory. These codes may be useful for whom already know the theory and want to revise these codes according to his work.

Ü. Ufuktepe and S. Kapçak give Mathematica modules for one- dimensional stability [2]. In that study they provide Mathematica applications including the stability of the one dimensional system, the Cobweb diagram for one dimensional system, the time series diagram, bifurcation diagrams and the phase plane diagrams for two-dimensional systems.

With this work we focus on only the "Stability Theory". We give the different codes for one dimensional models and two dimensional stability with Center Manifold Theory.

2. Stability Analysis for One Dimensional Models. Let $f: R \rightarrow R$ be a map where $R$ is the set of real numbers. Take an initial point $x_{0} \in \mathrm{R}$ and iterate this point with respect to the map $\mathrm{f}$. The set of values found with iterations give us the orbit $\mathrm{O}\left(x_{0}\right)$ of the point $x_{0}$. That is $\mathrm{O}\left(x_{0}\right)=\left\{x_{0}, \mathrm{f}\left(x_{0}\right), f^{2}\left(x_{0}\right), f^{3}\left(x_{0}\right), \ldots\right\}$ where $f^{n}=$ fofofo...f (n times)

If we let $x_{n}=f^{n}\left(x_{0}\right)$, then we obtain the first-order difference equation $x_{n+1}=\mathrm{f}\left(x_{n}\right)$

Definition (Fixed Point) : Consider the difference equation $x_{n+1}=\mathrm{f}\left(x_{n}\right)$

A point $x^{*}$ is said to be a fixed point of the map $f$ if $f\left(x^{*}\right)=x^{*}$

Definition (Stability): Let $f: I \rightarrow I$ be a map and $x^{*}$ be a fixed point of $f$, where $I$ is an interval in the set of real numbers $\mathrm{R}$. Then $\mathrm{x}^{*}$ is said to be stable if for any $\varepsilon>0$, there exists $\delta>0$ such that for all $x_{0} \in \mathrm{I}$ with $\left|x_{0}-\mathrm{x}^{*}\right|<\delta$ we have $\mid f^{n}\left(x_{0}\right)$ - $\mathrm{x}^{*} \mid<\varepsilon$ for all $\mathrm{n} \epsilon Z^{+}$.

Theorem (Criteria for Stability -for Hyperbolic Fixed Points): Let f be a map which is continuously differentiable at $x^{*}$. And let $x^{*}$ be a hyperbolic fixed point of the map $f$ that is $\left|f^{\prime}(x *)\right| \neq 1$. Then

1.If $\left|f^{\prime}(x *)\right|<1$, then $\mathrm{x}^{*}$ is asymptotically stable.

2.If $\left|f^{\prime}(x *)\right|>1$, then $\mathrm{x}^{*}$ is unstable.

The following code works when the user give a function $\mathrm{g}$ that depends on $\mathrm{x}$ and the output will be the fixed points with their types (stable-unstable).

OneDimensionalStability $\left[\mathrm{g}_{-}, \mathrm{x}_{-}\right]:=\operatorname{Module}[\{G, u, l, \mathrm{czl}, \mathrm{czl} 1, \mathrm{Sch}\}$,

$G\left[\mathrm{u}_{-}\right]:=g / . x \rightarrow u ; \operatorname{czl}=\operatorname{Solve}[G[u]==u, u] ; \operatorname{czl} 1=u / . \operatorname{czl} ; l=\operatorname{Length}[\operatorname{czl} 11]$

$\operatorname{Sch}\left[\mathrm{u}_{]}\right]:=G^{\prime \prime \prime}[u] / G^{\prime}[u]-3 / 2\left(G^{\prime \prime}[u] / G^{\prime}[u]\right)^{\wedge} 2$

Do[Which[

$-1<G^{\prime}[\operatorname{czl1}[[i]]]<1$, Print ["f has hyperbolic stable fixed point at ", "x=", czl1[[i]]],

$G^{\prime}[\operatorname{czl1}[[i]]]>1$, Print["f has hyperbolic unstable fixed point at ", "x=", czl1[[i]]],

$\left.G^{\prime}[\operatorname{czl1} 1[i]]\right]<-1$, Print ["f has hyperbolic unstable fixed point at ", "x=", czl1[[i]]],

$G^{\prime}[\operatorname{czl} 1[[i]]]==1$, If $\left[G^{\prime \prime}[\operatorname{czl} 1[[i]]] !=0\right.$,

Print["f has nonhyperbolic unstable (semistable) fixed point at", "x=", czl1[[i]]],

Which $\left[G^{\prime \prime \prime}[\operatorname{czl1}[[i]]]<0\right.$, Print[ "f has nonhyperbolic stable fixed point at ", " $\mathrm{x}=$ ", $\left.\operatorname{czl1}[[i]]\right]$,

$G^{\prime \prime \prime}[\operatorname{czl1}[[i]]]>0$, Print["f has nonhyperbolic unstable fixed point at ", "x=", czl1[[i]]]]],

$G^{\prime}[\operatorname{czl} 1[[i]]]==-1$, Which $[\operatorname{Sch}[\operatorname{czl} 1[[i]]]<0$,

Print ["f has nonhyperbolic stable fixed point at ", " $\mathrm{x}=$ ", $\operatorname{czl1}[[i]]], \operatorname{Sch}[\operatorname{czl1}[[i]]]>0$,

Print["f has nonhyperbolic unstable fixed point at ", "x=", czl1[[i]]]]], $\{i, 1, l\}]]$ 
Example 2.1. Let us find the fixed points of this one dimensional system $x_{n+1}=x_{n}^{2}+3 x_{n}$

In[1]:OneDimensionalStability $\left[x^{\wedge} 2+3 x, x\right]$

Out[1]:f has nonhyperbolic stable fixed point at $\mathrm{x}=-2$

$\mathrm{f}$ has hyperbolic unstable fixed point at $\mathrm{x}=0$

3. Stability Analysis for Two Dimensional Models. Let's consider the following linear systems:

$$
\begin{aligned}
& x_{n+1}=\mathrm{a} x_{n}+\mathrm{b} y_{n} \\
& x_{n+1}=\mathrm{c} x_{n}+\mathrm{d} y_{n}
\end{aligned}
$$

where a,b,c,d are real constants.

The only fixed point of this system is $(0,0)$. To investigate the stability of two dimensional linear maps, we need to find the eigenvalues of the coefficient matrix. According to eigenvalues of the linear system, one can determine the stability type of the fixed point $(0,0)$ :

If $0<\lambda_{1}<1<\lambda_{2}$ then saddle,

If $0<\lambda_{2}<\lambda_{1}<1$ then sink,

If $\lambda_{2}>\lambda_{1}>1$ then source,

If $\lambda=a+i b,|\lambda|<1, b \neq 0$ then spiral sink

If $\lambda=a+i b,|\lambda|>1, b \neq 0$ then spiral source

If $\lambda=a+i b,|\lambda|=1, b \neq 0$ then center

If $-1<\lambda_{1}<0, \lambda_{2}<-1$ then oscillatory saddle

If $\lambda_{1}>1, \lambda_{2}<-1$ then oscillatory source

Linear models are generally unrealistic for real world applications. Interactions do not allow to be linear. Ignoring these relations makes the model simpler but less reliable. So for a dynamical system being linear with no interactions is a strong condition and generally does not hold. At this point to say something about the stability of fixed points, linearization methods should be used. Linearization refers to finding the linear approximation to a function at a given point. In the study of dynamical systems, linearization is a method for assessing the local stability of a fixed point of a system of nonlinear discrete dynamical systems. For the stability analysis of nonlinear models, one can use the eigenvalues of the Jacobian matrix evaluated at the fixed point to determine the nature of that fixed point. Here Jacobian matrix can be thought as coefficient matrix in linear case. This linearization can be done for the neighborhood of a point.

Consider the following system where $\mathrm{f}$ and $\mathrm{g}$ are nonlinear.

$$
\begin{aligned}
& x_{n+1}=\mathrm{f}(\mathrm{x}, \mathrm{y}) \\
& y_{n+1}=\mathrm{g}(\mathrm{x}, \mathrm{y})
\end{aligned}
$$

The following module is used to find the fixed points and their stability of two dimensional system (for both linear and nonlinear cases). The user enters functions $f$ and $g$, the output gives the fixed points and their stability.

TwoDimensionalStability[f, $\left.\mathrm{f}_{-}\right]:=\operatorname{Module}[\{\mathrm{cz}, \mathrm{czm}, F, G, J, A, l\}$, $\mathrm{cz}=\operatorname{Solve}[\{f==x, g==y\},\{x, y\}] ; \operatorname{czm}=\{x, y\} / . \operatorname{cz} ; F\left[\mathrm{u}_{-}, \mathrm{v}_{-}\right]:=f / . x \rightarrow u$

$/ . y \rightarrow v ; G\left[\mathrm{u}_{-}, \mathrm{v}_{-}\right]:=g / . x \rightarrow u / . y \rightarrow v$;

$J\left[\left\{\mathrm{u}_{-}, \mathrm{v}_{-}\right\}\right]=D[\{F[u, v], G[u, v]\},\{\{u, v\}\}] ; l=\operatorname{Length}[\mathrm{czm}] ;$

If $[l==0, \operatorname{Print}[$ [No fixed point"], Do[If[Im[First[Eigenvalues[ $J[\operatorname{czm}[[i]]]]]]==0$,

If $[-1<\operatorname{Max}[$ Eigenvalues[$[J[\operatorname{czm}[[i]]]]]<1 \& \&-1<\operatorname{Min}[$ Eigenvalues $[J[\operatorname{czm}[[i]]]]]<1$,

Print["The fixed point" , $\operatorname{czm}[[i]]$, "is Stable sink"],

Which $[\operatorname{Max}[$ Eigenvalues $[J[\operatorname{czm}[[i]]]]]>1 \& \& 0<\operatorname{Min}[$ Eigenvalues $[J[\operatorname{czm}[[i]]]]]<1$,

Print: "The fixed point", $\operatorname{czm}[[i]]$, " is saddle"],

$-1<\operatorname{Max}[$ Eigenvalues $[J[\operatorname{czm}[[i]]]]]<0 \& \& \operatorname{Min}[$ Eigenvalues$[J[\operatorname{czm}[[i]]]]]<-1$,

Print ["The fixed point", $\operatorname{czm}[[i]]$, "is oscillatory saddle"], 
$\operatorname{Min}[$ Eigenvalues $[J[\operatorname{czm}[[i]]]]]>1$, Print ["The fixed point", $\operatorname{czm}[[i]]$, "is source"],

$\operatorname{Min}[$ Eigenvalues $[J[\operatorname{czm}[[i]]]]]==-1 \& \& \operatorname{Max}[$ Eigenvalues $[J[\operatorname{czm}[[i]]]]]<1$,

Print ["Try to use Center manifold module for ", $\operatorname{czm}[[i]]]$,

$\operatorname{Min}[$ Eigenvalues $[J[\operatorname{czm}[[i]]]]]==1$, Print ["The fixed point", $\operatorname{czm}[[i]]$, "is unstable"],

Max[Eigenvalues $[J[\operatorname{czm}[[i]]]]]==-1$, Print[ "The fixed point", $\operatorname{czm}[[i]]$, "is unstable"],

$\operatorname{Max}[$ Eigenvalues $[J[\operatorname{czm}[[i]]]]]==1 \& \& \operatorname{Min}[$ Eigenvalues $[J[\operatorname{czm}[[i]]]]]<-1$,

Print["The fixed point", czm[[i]], "is unstable"],

$\operatorname{Min}[$ Eigenvalues $[J[\operatorname{czm}[[i]]]]]==-1 \& \& \operatorname{Max}[$ Eigenvalues $[J[\operatorname{czm}[[i]]]]]>1$,

Print["The fixed point", czm[[i]], "is unstable"],

$\operatorname{Max}[$ Eigenvalues $[J[\operatorname{czm}[[i]]]]]==1 \& \& \operatorname{Min}[$ Eigenvalues $[J[\operatorname{czm}[[i]]]]]>-1$,

Print ["Try to use Center manifold module for ", czm[[i]]],

$\operatorname{Max}[$ Eigenvalues $[J[\operatorname{czm}[[i]]]]]>1 \& \& \operatorname{Min}[$ Eigenvalues $[J[\operatorname{czm}[[i]]]]]<-1$,

Print["The fixed point", czm[[i]] , " is oscillatory source"]]],

Which[Abs[First[Eigenvalues[ $J[\operatorname{czm}[[i]]]]]]<1$, Print["The fixed point", czm[[i]]],

" is spiral sink"], $\mathrm{Abs}[$ First [Eigenvalues[ $J[\operatorname{czm}[[i]]]]]]==1$, Print [

"The fixed point", $\operatorname{czm}[[i]]$, "is Center"], Abs[First[Eigenvalues $[J[\operatorname{czm}[[i]]]]]]>1$,

Print["The fixed point", $\operatorname{czm}[[i]]$, " is spiral source"]]], $\{i, 1, l\}]]]$

Example 3.1. Let's consider the following nonlinear systems

$$
\begin{aligned}
& x_{n+1}=x_{n}^{2}+3 y_{n} x_{n} \\
& y_{n+1}=y_{n}^{2}-2 y_{n} x_{n}
\end{aligned}
$$

If we write $f$ and $g$ in our module we get the fixed points and their stability.

In[2]:TwoDimensionalStability $\left[x^{\wedge} 2+3 y x, y^{\wedge} 2-2 y x\right]$

Out[2]:The fixed point $\left\{-\frac{2}{7}, \frac{3}{7}\right\}$ is saddle

The fixed point $\{0,1\}$ is source

The fixed point $\{1,0\}$ is oscillatory source

The fixed point $\{0,0\}$ is Stable sink

4. Center Manifolds. In two dimensional maps stability types are determined according to eigenvalues after linearization. If the fixed point is hyperbolic that is, $\left|\lambda_{i}\right| \neq 1 \mathrm{i}=1,2$, then it is simple. If at least one of them is greater than 1 or less than -1 , we can conclude that it is unstable. But the case $\left|\lambda_{1}\right|=1$ and $\left|\lambda_{2}\right|<1$ is more complicated case, it can be stable or unstable. If a nonhyperbolic map is defined on $R^{2}$, then the dynamics may be analyzed by studying the dynamics on an associated one- dimensional center manifold $M_{c}$. In mathematics, the center manifold of a fixed point of a dynamical system consists of orbits whose behavior around the fixed point is not controlled by either the attraction of the stable manifold or the repulsion of the unstable manifold.

First let us write our system in the following way:

$$
\begin{aligned}
& x_{n+1}=\mathrm{A} x_{n}+\mathrm{f}\left(x_{n}, y_{n}\right) \\
& y_{n+1}=\mathrm{B} y_{n}+\mathrm{g}\left(x_{n}, y_{n}\right)
\end{aligned}
$$

Since we interested in the fixed point write it as

$$
\begin{aligned}
& x \longrightarrow A x+f(x, y) \\
& y \longrightarrow B y+g(x, y)
\end{aligned}
$$

where $\mathrm{f}$ and $\mathrm{g}$ satisfies the following

$\mathrm{f}(0,0)=0, \mathrm{~g}(0,0)=0$

$\operatorname{Df}(0,0)=0, \operatorname{Dg}(0,0)=0$ 
Theorem: There is a $C^{r}$ center manifold for the system

that can be represented locally as

$$
\begin{aligned}
& \mathrm{x} \longrightarrow \mathrm{A} x+\mathrm{f}(\mathrm{x}, \mathrm{y}) \\
& \mathrm{y} \longrightarrow \mathrm{B} y+\mathrm{g}(\mathrm{x}, \mathrm{y})
\end{aligned}
$$

$M_{c}=\left\{(\mathrm{x}, \mathrm{y}) \epsilon R^{t} \mathrm{x} R^{s}: \mathrm{y}=\mathrm{h}(\mathrm{x}),|\mathrm{x}|<\delta, \mathrm{h}(0)=0, \operatorname{Dh}(0)=0\right.$, for sufficiently small $\left.\delta\right\}$

Furthermore, the dynamics restricted to $M_{c}$ are given locally by the map [1]

$$
\mathrm{x} \longrightarrow \mathrm{A} \mathrm{x}+\mathrm{f}(\mathrm{x}, \mathrm{h}(\mathrm{x})), \quad \mathrm{x} \in R^{t}
$$

Theorem: If the fixed point 0 of the equation $\mathrm{x} \longrightarrow \mathrm{Ax}+\mathrm{f}(\mathrm{x}, \mathrm{h}(\mathrm{x}))$ is stable, asymptotically stable, or unstable then the fixed point $(0,0)$ of the system

$$
\begin{aligned}
& \mathrm{x} \longrightarrow \mathrm{A} x+\mathrm{f}(\mathrm{x}, \mathrm{y}) \\
& \mathrm{y} \longrightarrow \mathrm{B} \mathrm{y}+\mathrm{g}(\mathrm{x}, \mathrm{y})
\end{aligned}
$$

is also stable, asymptotically stable, or unstable [1].

The important question is how to compute the center manifold or equivalently the curve $\mathrm{y}=\mathrm{h}(\mathrm{x})$. Consider again the system

$$
\begin{aligned}
& x_{n+1}=A x_{n}+f\left(x_{n}, y_{n}\right) \\
& y_{n+1}=B y_{n}+g\left(x_{n}, y_{n}\right)
\end{aligned}
$$

By plugging $y_{n}=h\left(x_{n}\right)$ into (1) we get the following equation:

$$
y_{n+1}=\mathrm{h}\left(x_{n+1}\right)=\mathrm{h}\left(\mathrm{A} x_{n}+\mathrm{f}\left(x_{n}, \mathrm{~h}\left(x_{n}\right)\right)=\mathrm{B} \mathrm{h}\left(x_{n}\right)+\mathrm{g}\left(x_{n}, \mathrm{~h}\left(x_{n}\right)\right)\right.
$$

By solving the system we get the following functional equation:

$$
h[A x+f(x, h(x))]-B h(x)-g(x, h(x))=0
$$

It is still a problem to find $\mathrm{h}(\mathrm{x})$. Since we have $\mathrm{h}(0)=0, \mathrm{Dh}(0)=0$, by Taylor Expansion $h(x)=c_{1} x^{2}+c_{2} x^{3}+O\left(x^{4}\right)$. Note that the Center Manifold Theorem can be used only for the fixed point $(0,0)$. Otherwise, the fixed point must be transformed to $(0,0)$.

If the system is in the form $\mathrm{x} \longrightarrow \mathrm{A} \mathrm{x}+\mathrm{f}(\mathrm{x}, \mathrm{y})$ and $\mathrm{y} \longrightarrow \mathrm{B} \mathrm{y}+\mathrm{g}(\mathrm{x}, \mathrm{y})$, then the user can use the following module to find the center manifold curve $h$, and type of the system. The user should enter the values $\mathrm{A}$ and $\mathrm{B}$, the functions $\mathrm{f}$ and $\mathrm{g}$.

CenterManifold1[A, B_, f, $\left._{-}, g_{-}\right]:=$

Module $\left[\{h, S, F, G, K, T\right.$, coef, $\mathrm{c} 1, \mathrm{c} 2, \mathrm{f} 1, \mathrm{f} 2, \mathrm{Sch}\}, F\left[\mathrm{u}_{-}, \mathrm{v}_{-}\right]:=f / . x \rightarrow u$

$/ . y \rightarrow v ; G\left[\mathrm{u}_{-}, \mathrm{v}_{-}\right]:=g / . x \rightarrow u / . y \rightarrow v ; h\left[\mathrm{u}_{-}, \mathrm{c}_{-}, \mathrm{d}_{-}\right]:=c u^{\wedge} 2+d u^{\wedge} 3+O[u]^{\wedge} 4$;

$S=$ ForAll $[u, h[A u+F[u, h[u, c, d]], c, d]==B h[u, c, d]+G[u, h[u, c, d]]]$

$K=\operatorname{Resolve}[S$, Reals]; $T=$ Solve $[K,\{c, d\}] ; \operatorname{coef}=$ Flatten $[\{c, d\} / . T]$;

$\mathrm{c} 1=$ First[coef]; $\mathrm{c} 2=$ Last[coef]; f1 $=A u+F[u, h[u, \mathrm{c} 1, \mathrm{c} 2]] ; \mathrm{f} 2\left[\mathrm{a}_{-}\right]:=\mathrm{f} 1 / . u \rightarrow a$;

$\operatorname{Print}[" \mathrm{~h}(\mathrm{x})=", h[x, \mathrm{c} 1, \mathrm{c} 2]]$;

Print["The map $\mathrm{f}$ on the center manifold is given by $\mathrm{x}->$ ", $\mathrm{f} 2[x]]$;

$\mathrm{Sch}\left[\mathrm{a}_{-}\right]=\mathrm{f} 2^{\prime \prime \prime}[a] / \mathrm{f} 2^{\prime}[a]-3 / 2\left(\mathrm{f} 2^{\prime \prime}[a] / \mathrm{f} 2^{\prime}[a]\right)^{\wedge} 2$;

Which $\left[-1<\mathrm{f}^{\prime}[0]<1\right.$, Print["The fixed point $(0,0)$ is stable"],

$\mathrm{f}^{\prime}[0]>1$, Print ["The fixed point $(0,0)$ is unstable "],

f2' $[0]<-1$, Print["The fixed point $(0,0)$ is unstable"],

$\mathrm{f}^{\prime}[0]==1$,

If $[\mathrm{f} 2 "[0] !=0$, Print ["The fixed point $(0,0)$ is unstable"], If[f2 2 "' $[0]<0$,

Print ["The fixed point $(0,0)$ is stable"], Print ["The fixed point $(0,0)$ is unstable"]],

$\mathrm{f}^{\prime}[0]==-1$, If $[\operatorname{Sch}[0]<0$, Print [ "The fixed point $(0,0)$ is stable"],

Print["The fixed point $(0,0)$ is unstable"]]]] 
Example 4.1. Let's consider the following non-linear discrete dynamical systems

$$
\begin{aligned}
& x_{n+1}=-x_{n}+5 x_{n} y_{n} \\
& y_{n+1}=-\frac{1}{4} y_{n}+3 x_{n}^{2}
\end{aligned}
$$

If we write $\mathrm{f}$ and $\mathrm{g}$ in our TwoDimensionalStability module we get the fixed points and their stability.

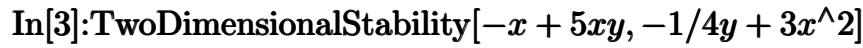

Out[3]:Try to use Center manifold module for $\{0,0\}$

The fixed point $\left\{-\frac{1}{\sqrt{6}}, \frac{2}{5}\right\}$ is oscillatory source

The fixed point $\left\{\frac{1}{\sqrt{6}}, \frac{2}{5}\right\}$ is oscillatory source

To find the stability of $(0,0)$ the CenterManifold1 module is used. If we write the values A and $\mathrm{B}$, the functions $\mathrm{f}$ and $\mathrm{g}$, the module gives the stability of $(0,0)$

$\operatorname{In}[4]:$ CenterManifold1 $\left[-1,-1 / 4,5 x y, 3 x^{\wedge} 2\right]$

Out $[4]: \mathrm{h}(\mathrm{x})=\frac{12 x^{2}}{5}+O[x]^{4}$

The map $\mathrm{f}$ on the center manifold is $\mathrm{x}->-x+12 x^{3}+O[x]^{5}$

The fixed point $(0,0)$ is stable

Example 4.2. Let's consider the following non-linear discrete dynamical systems

$$
\begin{aligned}
& x_{n+1}=-x_{n}+x_{n} y_{n} \\
& y_{n+1}=\frac{1}{2} y_{n}+\frac{1}{2} x_{n}^{2}+2 x^{2} y+y^{3}
\end{aligned}
$$

If we write $\mathrm{f}$ and $\mathrm{g}$ in our TwoDimensionalStability module we get the fixed points and their stability.

$\operatorname{In}[5]:$ TwoDimensionalStability $\left[x+x y,(1 / 2 y)+(1 / 2) x^{\wedge} 2+2\left(x^{\wedge} 2\right) y+y^{\wedge} 3\right]$

Out[5]:Try to use Center manifold module for $\{0,0\}$

The fixed point $\left\{0,-\frac{1}{\sqrt{2}}\right\}$ is saddle

The fixed point $\left\{0, \frac{1}{\sqrt{2}}\right\}$ is source

If we write the values $\mathrm{A}$ and $\mathrm{B}$, the functions $\mathrm{f}$ and $\mathrm{g}$, the CenterManifold 1 module gives the stability of $(0,0)$

$\operatorname{In}[6]:$ CenterManifold1 $\left[1,1 / 2, x y,(1 / 2) x^{\wedge} 2+2\left(x^{\wedge} 2\right) y+y^{\wedge} 3\right]$

Out $[6]: \mathrm{h}(\mathrm{x})=x^{2}+O[x]^{4}$

The map $\mathrm{f}$ on the center manifold is given by $\mathrm{x}->x+x^{3}+O[x]^{5}$

The fixed point $(0,0)$ is unstable

The module CenterManifold1 is for the models

$\mathrm{x} \longrightarrow \mathrm{Ax}+\mathrm{f}(\mathrm{x}, \mathrm{y})$

$\mathrm{y} \longrightarrow \mathrm{By}+\mathrm{g}(\mathrm{x}, \mathrm{y})$ that is

$\left(\begin{array}{l}x \\ y\end{array}\right) \longrightarrow \mathrm{J} .\left(\begin{array}{l}x \\ y\end{array}\right)+\left(\begin{array}{l}f \\ g\end{array}\right) \quad$ where $\mathrm{J}=\left(\begin{array}{cc}A & 0 \\ 0 & B\end{array}\right)$

If $\mathrm{J}$ is not a diagonal matrix, then we use diagonalization by using matrix $\mathrm{T}$ whose columns are the eigenvectors $V_{1}$ and $V_{2}$

Letting $\left(\begin{array}{l}x \\ y\end{array}\right)=\mathrm{T} \cdot\left(\begin{array}{l}u \\ v\end{array}\right)$ 
$\mathrm{T}\left(\begin{array}{l}u \\ v\end{array}\right) \longrightarrow \mathrm{J} . \mathrm{T}\left(\begin{array}{l}u \\ v\end{array}\right)+\left(\begin{array}{l}f \\ g\end{array}\right)$

$\left(\begin{array}{c}u \\ v\end{array}\right) \longrightarrow T^{-1} \mathrm{~J} . T\left(\begin{array}{c}u \\ v\end{array}\right)+T^{-1}\left(\begin{array}{c}f \\ g\end{array}\right)$ where $\mathrm{f}$ and $\mathrm{g}$ should be written in terms of $\mathrm{u}$ and $\mathrm{v}$.

The following module can be used for $\left(\begin{array}{l}x \\ y\end{array}\right) \rightarrow \mathrm{J} .\left(\begin{array}{l}x \\ y\end{array}\right)+\left(\begin{array}{l}f \\ g\end{array}\right)$ type systems where $\mathrm{J}$ is not diagonal.

The user will give the jacobian matrix and $\left(\begin{array}{c}f \\ g\end{array}\right)$. Note that both of them should be in the matrix form. The output is center manifold curve $h$, the corresponding one dimensional map and stability of $(0,0)$.

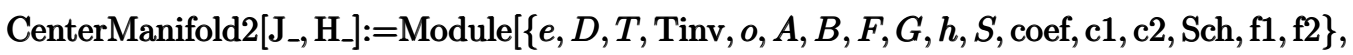
$e=$ Eigenvalues $[J] ; D=\{\{e[[1]], 0\},\{0, e[[2]]\}\} ; T=$ Transpose[Eigenvectors $[J]]$;

$\{\{x\},\{y\}\}=T .\{\{u\},\{v\}\} ; \operatorname{Tinv}=\operatorname{Inverse}[T] ; o=$ Tinv. $H ; A=e[[1]] ; B=e[[2]] ;$

$F\left[\mathrm{z}_{-}, \mathrm{p}_{-}\right]=o[[1,1]] / . u \rightarrow z$

$/ . v \rightarrow p ; G\left[\mathrm{z}_{-}, \mathrm{p}_{-}\right]=o[[2,1]] / . u \rightarrow z$

$/ . v \rightarrow p ; h\left[\mathrm{z}_{-}, \mathrm{c}_{-}, \mathrm{d}_{-}\right]:=c z^{\wedge} 2+d z^{\wedge} 3+O[z]^{\wedge} 4$;

$S=\operatorname{Solve}[h[A z+F[z, h[z, c, d]], c, d]==B h[z, c, d]+G[z, h[z, c, d]],\{c, d\}]$

coef $=$ Flatten $[\{c, d\} / . S] ;$ c1 = First [coef $] ;$ 2 = Last $[$ coef $] ;$

$\mathrm{f} 1=A z+F[z, h[z, \mathrm{c} 1, \mathrm{c} 2]] ; \mathrm{f} 2\left[\mathrm{a}_{-}\right]:=\mathrm{f} 1 / . z \rightarrow a ; \operatorname{Print}[$ "h(u) $=", h[u, \mathrm{c} 1, \mathrm{c} 2]]$

Print ["The map $\mathrm{f}$ on the center manifold is given by $\mathrm{u}->$ ", $\mathrm{f} 2[u]]$;

$\operatorname{Sch}\left[\mathrm{a}_{-}\right]=\mathrm{f2} 2^{\prime \prime \prime}[a] / \mathrm{f2} 2^{\prime}[a]-3 / 2\left(\mathrm{f2} 2^{\prime \prime}[a] / \mathrm{f2}^{\prime}[a]\right)^{\wedge} 2$;

Which $\left[-1<\mathrm{f}^{\prime}[0]<1\right.$, Print ["The fixed point $(0,0)$ is stable"],

f2' $[0]>1$, Print ["The fixed point $(0,0)$ is unstable "],

f2' $[0]<-1$, Print ["The fixed point $(0,0)$ is unstable "],

$\mathrm{f}^{\prime}[0]==1$,

If[f2" $[0] !=0$, Print["The fixed point $(0,0)$ is unstable"], If[f2"' $[0]<0$,

Print["The fixed point $(0,0)$ is stable"], Print["The fixed point $(0,0)$ is unstable"]]],

$\mathrm{f2}^{\prime}[0]==-1, \mathrm{If}[\operatorname{Sch}[0]<0$, Print ["The fixed point $(0,0)$ is stable"],

Print["The fixed point $(0,0)$ is unstable"]]]]

Example 4.3. Let's consider the following non-linear discrete dynamical systems

$$
\begin{aligned}
& x_{n+1}=y_{n} \\
& y_{n+1}=-\frac{1}{4} x_{n}+\frac{5}{4} y_{n}-2 y^{3}
\end{aligned}
$$

If we write $\mathrm{f}$ and $\mathrm{g}$ in our TwoDimensionalStability module we get the fixed points and their stability.

$\operatorname{In}[7]:$ TwoDimensionalStability $\left[\left[y,(-1 / 4) x+(5 / 4) y-2 y^{\wedge} 3\right]\right.$

Out[7]:Try to use Center manifold module for $\{0,0\}$

If we write the Jacobian matrix and $\left(\begin{array}{c}f \\ g\end{array}\right)$ CenterManifold2 module gives the stability of $(0,0)$ In[8]:CenterManifold2[\{\{0,1\}, $\left.\{-1 / 4,5 / 4\}\},\left\{\{0\},\left\{-2 y^{\wedge} 3\right\}\right\}\right]$

Out $[8]: \mathrm{h}(\mathrm{u})=\frac{8 u^{3}}{9}+O[u]^{4}$

The map $\mathrm{f}$ on the center manifold is given by $\mathrm{u}->u-\frac{8 u^{3}}{3}-\frac{64 u^{5}}{9}+O[u]^{6}$

The fixed point $(0,0)$ is stable

CenterManifold2 is more general than CenterManifold1, so it can be used for diagonal case, too. 
Example 4.4. Let's consider Example 4.1 again.

$\operatorname{In}[9]:$ CenterManifold2[\{\{-1,0\}, $\left.\{0,-1 / 4\}\},\left\{\{5 x y\},\left\{3 x^{\wedge} 2\right\}\right\}\right]$

$\operatorname{Out}[9] \mathrm{h}(\mathrm{u})=\frac{12 u^{2}}{5}+O[u]^{4}$

The map $\mathrm{f}$ on the center manifold is given by $\mathrm{u}->-u+12 u^{3}+O[u]^{5}$

The fixed point $(0,0)$ is stable

5. Conclusion. In this work, we give the stability analysis of one and two dimensional dynamical models with Mathematica. We try to do the analysis for both hyperbolic and non-hyperbolic fixed points. We do not use complex language in the codes. So they are understandable and easily appliable. As a result, these codes may be useful not only for the researchers who want to apply them without studying the whole theory, but also for the researchers who want to develop these modules using the theory. Future works will be about Bifurcation theory for two dimensional discrete dynamical systems.

\section{REFERENCES}

[1] Elaydi, S. N. (2008). Discrete Chaos: With Applications in Science and Engineering. CRC Press.

[2] Ufuktepe, Ü., \& Kapçak, S (2013). Applications of Discrete Dynamical Systems with Mathematica.

[3] S. Wolfram,(1996) The Mathematica Book, Wolfram Media/Cambridge University Press, ThirdEdition. 